\title{
Unexpected extrusion of the implantable pulse generator of the spinal cord stimulator - A case report -
}

\section{Eun-Ji Choi ${ }^{1,2}$, Hyun-Su Ri ${ }^{1,2}$, Hyeonsoo Park ${ }^{1}$, Hye-Jin Kim ${ }^{1,2}$, Ji-Uk Yoon ${ }^{1,2}$, and Gyeong-Jo Byeon ${ }^{1,2}$}

'Department of Anesthesia and Pain Medicine, Pusan National University Yangsan Hospital, Pusan National University School of Medicine, ${ }^{2}$ Research Institute for Convergence of Biomedical Science and Technology, Pusan National University Yangsan Hospital, Yangsan, Korea
Received June 23, 2020

Revised October 4, 2020

Accepted November 26, 2020

\section{Corresponding author}

Gyeong-Jo Byeon, M.D., Ph.D.

Department of Anesthesia and Pain Medicine, Pusan National University

Yangsan Hospital, Pusan National University School of Medicine, Geumoro 20, Mulgeumeup, Yangsan 50612, Korea

Tel: 82-55-360-2758

Fax: 82-55-360-2149

E-mail: byeongj@pusan.ac.kr
Background: Despite significant technological advances in the implantable pulse generator (IPG), complications can still occur. We report a case that unexpected extrusion of the IPG of spinal cord stimulation (SCS) was promptly identified and successfully removed without any complications.

Case: After a car accident 4 years ago, a 55-year-old male who was diagnosed with complex local pain syndrome in his right leg. The SCS was inserted with 2 leads, with the IPG being implanted in the right lower abdomen region. Four years later, he developed extrusion of the IPG from his abdominal region. This unexpected extrusion may have been related to pressure necrosis caused by continued compression of pocket site where a belt was frequently tied. The IPG and the leads were successfully removed without infection occurring.

Conclusions: To prevent unexpected extrusion of IPG, it is necessary to consider in advance whether the pocket site is pressed against the belt.

Keywords: Complex regional pain syndrome; Devices; Necrosis; Neuropathic pain; Spinal cord stimulation.
Spinal cord stimulation (SCS) has been used to treat various chronic neuropathic pain conditions for many decades [1]. SCS has been reported to be a relatively safe and reversible procedure with several complications due to minimally invasive properties. Common complications associated with SCS include lead migration, connection failure, lead breakage, pain at the implant site, seroma formation, and infection [2]. Catastrophic complications, including breakdown of the tissue overlaying implant site and extrusion of the device through the skin are possible, but very rare [3]. Pacemakers, which have a structure similar to that of the implantable pulse generator (IPG) component, have been reported to extrude out of the chest [4].

We report a case that unexpected extrusion of the IPG of SCS was promptly identified and successfully removed without any further adverse complications. Prior to this report, we received prior written informed consent for publication from the patient.

This is an Open Access article distributed under the terms of the Creative Commons Attribution Non-Commercial License (http://creativecommons.org/licenses/by-nc/4.0) which permits unrestricted non-commercial use, distribution, and reproduction in any medium, provided the original work is properly cited.

Copyright (C) the Korean Society of Anesthesiologists, 2021 


\section{CASE REPORT}

A 55-year-old male with a height of $169.2 \mathrm{~cm}$ and a weight of $71.5 \mathrm{~kg}$, who was diagnosed with complex regional pain syndrome in the right leg, was visited our pain clinic to evaluate SCS. He was in a car accident four years ago. There were no particular fractures at the time of the accident, and 3 months later, despite proper treatment, he suffered from refractory, persistent pain, edema, temperature and color changes, and hyperhidrosis of his right leg and foot. He was taking various oral medications (acetaminophen, tramadol, gabapentin, duloxetine, baclofen, and oxycodone) and had undergone several interventions (lumbar transforaminal epidural steroid injection, lumbar sympathetic ganglion block, and lumbar sympathetic alcohol neurolysis), but there was no significant pain relief. He complained that his baseline leg pain was 10 out of 10 in severity. The findings of magnetic resonance imaging, electromyography with nerve conduction study, bone densitometry, and 3-phase bone scan were non-specific. Digital infrared thermographic imaging of the lower extremities showed a body temperature $1-2.5^{\circ} \mathrm{C}$ lower in the right leg compared to the left leg.

Prior to the trial implantation of the SCS device, he conducted a structural interview and the Minnesota Multiphasic Personality Inventory by a psychologist. He was also trained about the system, its use, and the trial and implant procedures using printed materials and videos.

After he was admitted to our hospital and had agreed to the operation, he was offered an SCS trial using a lead delivery system device (Epiducer ${ }^{\mathrm{TM}}$, St. Jude Medical Neuromodulation Division, USA). After the skin was anesthetized, a 14-gauge needle, a steerable guide wire was inserted into the L4/L5 epidural space using fluoroscopy. The needle was then removed, and the Epiducer ${ }^{\mathrm{TM}}$ was threaded over the guidewire and into the epidural space. After finding no regurgitation of cerebrospinal fluid or blood, the inner dilator and guide wire are removed. The S-Series paddle lead (St. Jude Medical Neuromodulation Division) was inserted via the Epiducer ${ }^{\mathrm{rM}}$, and the lead tip was located at the T10 to T12 level. The test simulation was performed in a range of 2 to $1,200 \mathrm{~Hz}$, with typical pulse frequencies of 40 to $60 \mathrm{~Hz}$. Stimulation covered the patient's right leg and the dorsum of his foot. The lead was buried in the subcutaneous tissue. During the SCS trial, the pain decreased to 4 out of 10 . He reported greater than $50 \%$ relief of pain in his right leg. After 1 week of trial stimulation, the implantable pulse generator (IPG) was implanted. Before the operation, the implantation and incision sites were examined and marked in a sitting or standing position, the subcutaneous pocket site for the IPG (Proclame ${ }^{\mathrm{TM}}$, St. Jude Medical Neuromodulation Division) was made at the right lower abdominal wall. The IPG was implanted $2 \mathrm{~cm}$ deep in the right abdominal subcutaneous pocket site. The SCS was functioning well and showed no immediate post-surgical complications.

Two months later, he was generally satisfied with the pain relief; however, he reported that the stimulation did not come to the medial and sole of the foot, and hoped to reduce the pain in the region using another cylindrical lead (Octrode, St. Jude Medical Neuromodulation Division) for spinal cord stimulation. Therefore, we performed an additional operation to insert the lead, and the lead tip was located at the L1-L2 level (Fig. 1). He reported that electrical stimulation was smooth on all parts of his right leg and foot, and he experienced marked pain relief. The patient visited the clinic for follow-up evaluations every 1-2 months after the procedure. He was very satisfied with the pain relief on his right leg. In daily life, he paid special attention not to press the IPG insertion site when sleeping or

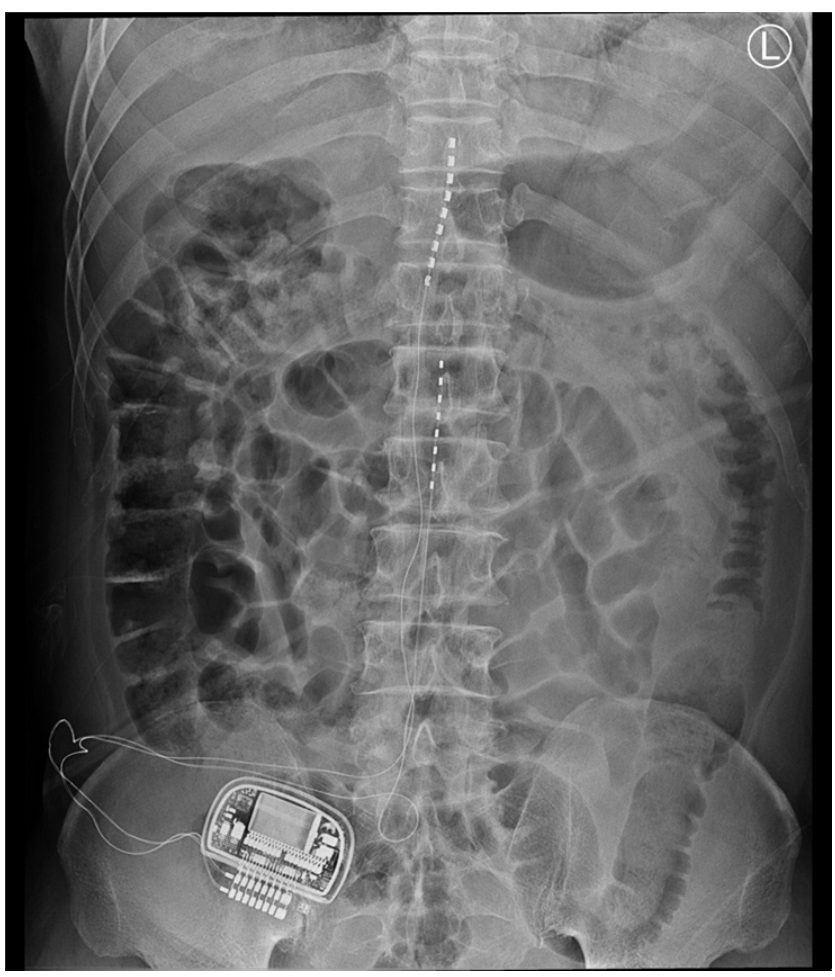

Fig. 1. The lead tips were located at the T10-T12 (paddle lead) and L1-L2 (cylindrical lead) level on simple X-ray L-spine anterior-posterior view. 
during activities, however, he was engaged in agriculture and was always doing hard work with his body bent in a sitting position. He occasionally noted a foreign body sensation in the IPG pocket site when wearing a belt. Sometimes, when he tightened his belt, he complained that the skin of the IPG site felt under pressure.

At a routine follow-up, he complained of wound site erosion, and a metal piece was exposed from his lower abdominal wall for 2 days. He denied having fevers and chills. On examination, the IPG had moved approximately $3 \mathrm{~cm}$ below the pocket, and a $0.5 \mathrm{~cm}$ area of metal part was extruding from his right lower abdominal wall. There was erosion around the entry and exit points of the IPG, with apparent healing of the tissue beneath. There was no discharge from the extrusion site from the eroded areas, and microbiological cultures were taken from several points around that region (Fig. 2). The pocket did not show any signs of active inflammation or infection. The IPG test revealed that the device's functional values were completely within normal ranges. After obtaining written informed consent, the patient had the stimulator leads and the IPG removed. The lower abdominal pocket site was thoroughly cleaned and debrided after the IPG removal, and the wound was sutured (Fig. 3). After surgery, the patient recovered uneventfully. The stimulator leads and the IPG removed during surgery were sent for aerobic and anaerobic culture, which returned back negative. The patient was prescribed cefazoline as postoperative antibiotic for 14 days. After removal of IPG, his right leg pain was 8-9 out of 10 in severity, as the pain was managed with only oral medication and several conservative interventions. With all the risk of infection removed and stable, we decided to

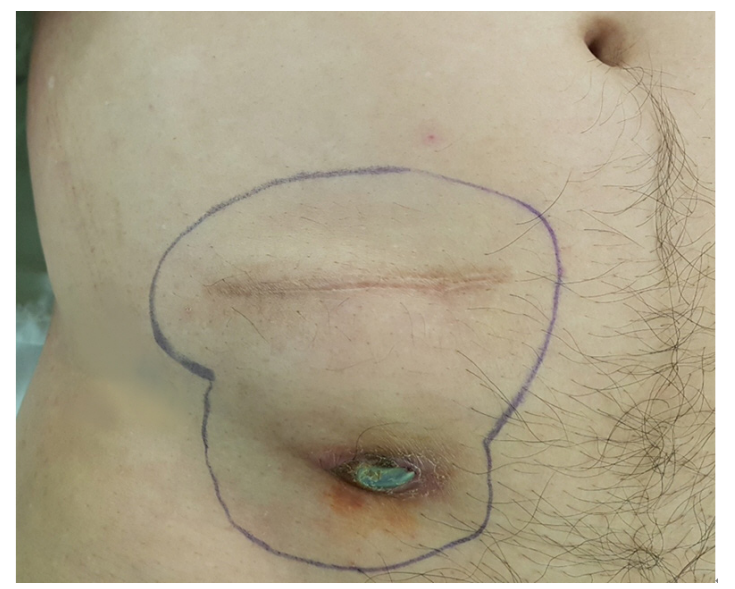

Fig. 2. The implantable pulse generator was extruded at the lower abdominal pocket site. perform re-implantation of the SCS device later.

\section{DISCUSSION}

SCS has been an effective surgical procedure for improving suffering among patients with chronic neuropathic pain. However, several complications can occur despite significant technological advances in the IPG, with significant decreases in both size and weight, and rechargeable capabilities.

Complications of SCS have been reported to have an incidence of $30-40 \%$ in several studies $[5,6]$. Its complications are divided into three main categories: hardware-related, biological, and programming or therapy-related. Hardware-related complications include lead fracture or disconnection reported incidence of 5-9\%, lead migration reported incidence of $0-27 \%$, and IPG failure in around $1.7 \%$ $[1,7]$. Biological complications include pain at the implant site, allergic reaction, IPG seroma, infection, epidural fibrosis, epidural hematoma, dura puncture-related headaches and more serious nerve damage, including spinal cord injury and paralysis $[8,9]$. Programming or therapy-related complications include loss of paresthesia and painful

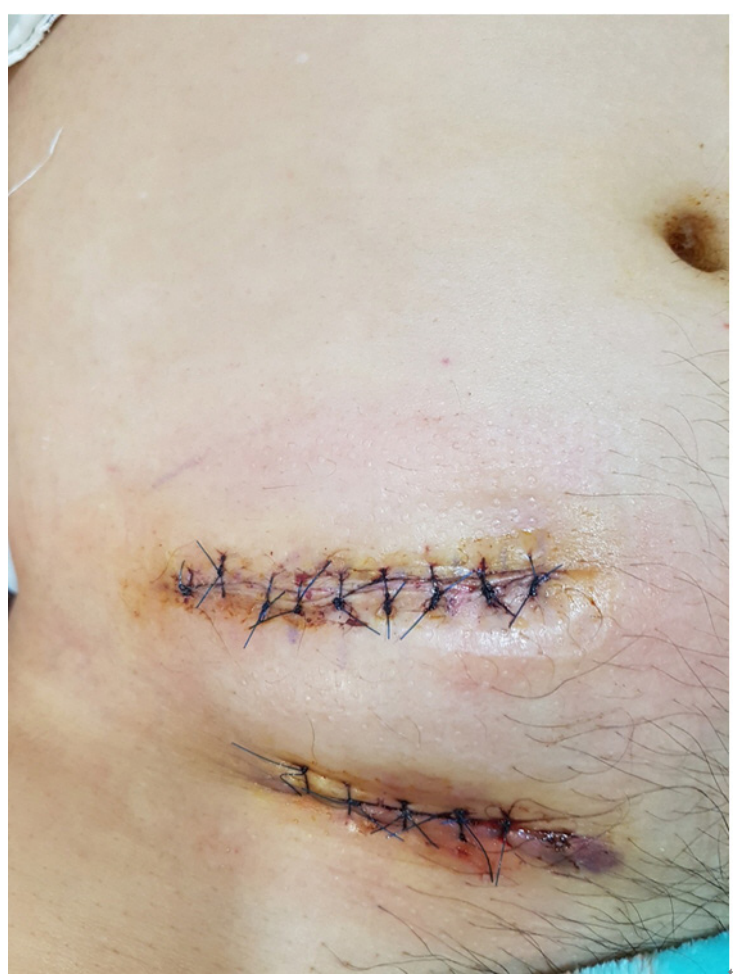

Fig. 3. The lower abdominal pocket site was thoroughly cleaned and debrided after implantable pulse generator removal, and the wound was sutured. 
or unpleasant paresthesia. These are less threatening and can usually be resolved through programming, although on rare occasions can be removed due to therapy failure [10].

Among the several complications mentioned above, IPG extrusion is a hardware-related complication, and it is a very rare complication of SCS. In two cases, IPG extrusion was reported after implantation in the gluteal area $[3,11]$. In one of these cases, the patient was a truck driver who had been driving for a long time for three months, causing skin erosion in the buttocks and extruding the IPG. In the other case, an extrusion of the IPG in a sacral stimulator was due to rapid weight loss after the patient underwent gastric bypass surgery. Our report is the first case of extrusion after implantation of the IPG of a spinal cord stimulator into the abdominal wall. In a case similar to our report, there are multiple case reports of pulse generator extrusion of a pacemaker $[4,12]$. They were caused by the skin eroding around the IPG insertion. The incidence of skin erosion due to the underlying pacemaker generator has been estimated to be approximately $0.8 \%$. Factors predisposing skin erosion are the presence of a thin subcutaneous fat layer, tissue fragility in old-age patients, abrasive action exerted on the skin from external agents, pressure exercised from the device on the subcutaneous tissue and possible infections of the site [13].

The patient had been doing well after the operation. Occasionally the patient complained of the discomfort of the IPG insertion when wearing a belt. The IPG were sutured to the subcutaneous fat layer of the abdominal wall to fix at the initial insertion site, but it seems that a tear occurred at the suture site. As a result, the IPG migrated downward from the initial insertion site, and it seems that the downward migration was a little worse due to the compression of the belt. Skin erosion occurred due to compression of the belt, and the IPG was extruded through this area. Whenever the patient wore a belt, he complained of discomfort due to the constant pressure on the IPG pocket site. We should have carefully observed skin erosion during follow-up. The early stages of skin erosion can develop exposure of the pocket site, and even IPG migration and extrusion. If the IPG size and configuration are not appropriate, excessive pressure can be placed on the subcutaneous tissue, and improperly sized pockets may result in the development of infection and dysfunction of the IPG [14]. With a careful follow-up as well as a clear understanding of potential complications and a careful approach to device selection can minimize the incidence of complications. It is important to identify early signs of erosion before the device damages the skin. If the skin is not damaged, surgical modification of the pocket is often necessary to prevent contamination and infection of the device. However, if the hardware is exposed, it should be assumed that the device is contaminated, and treatments generally involve a much more complicated procedure to remove all devices, including IPG and leads [15].

In conclusion, extrusion of the IPG from the pocket site is very rare. In order to prevent this rare complication, prior to permanent SCS insertion, the clinicians should fully consider the patient's age, occupational hazards, and daily life habits that could cause excessive pressure on the IPG. And the skin of abdominal wall should be inspected carefully at the site of the intended pocket, and the belt-tightening area should be examined in advance to see if all the SCS components, such as the IPG, will not be pressed by the belt. A deep pocket (about 2-3 cm depth), instead of just a pocket under the skin, should be considered. In addition, it is necessary to educate the patient not to habitually touch the IPG insertion site with a sense of foreign body.

\section{ACKNOWLEDGEMENTS}

This work was supported by clinical research grant from Pusan National University Yangsan Hospital in 2019.

\section{CONFLICTS OF INTEREST}

No potential conflict of interest relevant to this article was reported.

\section{AUTHOR CONTRIBUTIONS}

Conceptualization: Eun-Ji Choi, Gyeong-Jo Byeon. Writing - original draft: Eun-Ji Choi, Gyeong-Jo Byeon. Writing - review \& editing: Ji-Uk Yoon, Gyeong-Jo Byeon. Investigation: Hyun-Su Ri, Hyeonsoo Park, Hye-Jin Kim. Supervision: Ji-Uk Yoon.

\section{ORCID}

Eun-Ji Choi, https://orcid.org/0000-0003-3731-0785

Hyun-Su Ri, https://orcid.org/0000-0002-7305-4144

Hyeonsoo Park, https://orcid.org/0000-0002-3376-3691 
Hye-Jin Kim, https://orcid.org/0000-0003-1630-0422

Ji-Uk Yoon, https://orcid.org/0000-0002-3971-2502

Gyeong-Jo Byeon, https://orcid.org/0000-0001-5333-3894

\section{REFERENCES}

1. Deer TR, Mekhail N, Provenzano D, Pope J, Krames E, Leong M, et al. Neuromodulation Appropriateness Consensus Committee. The appropriate use of neurostimulation of the spinal cord and peripheral nervous system for the treatment of chronic pain and ischemic diseases: the Neuromodulation Appropriateness Consensus Committee. Neuromodulation 2014; 17: 515-50.

2. Mekhail NA, Mathews M, Nageeb F, Guirguis M, Mekhail MN, Cheng J. Retrospective review of 707 cases of spinal cord stimulation: indications and complications. Pain Pract 2011; 11: 148-53.

3. Rabi J, Anitescu M. Late extrusion of an implantable pulse generator of a spinal cord stimulator. Pain Physician 2016; 19: E671-4.

4. Yuksel S, Demir S, Sahin M. Total extrusion of a normally functioning pacemaker. Tex Heart Inst J 2012; 39: 156-7.

5. Kumar K, Taylor RS, Jacques L, Eldabe S, Meglio M, Molet J, et al. Spinal cord stimulation versus conventional medical management for neuropathic pain: a multicentre randomised controlled trial in patients with failed back surgery syndrome. Pain 2007; 132: 179-88.

6. Kemler MA, Barendse GA, van Kleef M, de Vet HC, Rijks CP, Furnée CA, et al. Spinal cord stimulation in patients with chronic reflex sympathetic dystrophy. N Engl J Med 2000;
343: 618-24.

7. Cameron T. Safety and efficacy of spinal cord stimulation for the treatment of chronic pain: a 20 -year literature review. J Neurosurg 2004; 100: 254-67.

8. Levy R, Henderson J, Slavin K, Simpson BA, Barolat G, Shipley J, et al. Incidence and avoidance of neurologic complications with paddle type spinal cord stimulation leads. Neuromodulation 2011; 14: 412-22.

9. Barolat G. Experience with 509 plate electrodes implanted epidurally from C1 to L1. Stereotact Funct Neurosurg 1993; 61: 60-79.

10. Kumar K, Wilson JR, Taylor RS, Gupta S. Complications of spinal cord stimulation, suggestions to improve outcome, and financial impact. J Neurosurg Spine 2006; 5: 191-203.

11. Nold CJ, McLennan MT. Spontaneous extrusion of sacral nerve implant secondary to massive weight loss. Int Urogynecol J Pelvic Floor Dysfunct 2007; 18: 105-7.

12. Santarpia G, Sarubbi B, D'Alto M, Romeo E, Calabro R. Extrusion of the device: a rare complication of the pacemaker implantation. J Cardiovasc Med (Hagerstown) 2009; 10: 330-2.

13. Harcombe AA, Newell SA, Ludman PF, Wistow TE, Sharples LD, Schofield PM, et al. Late complications following permanent pacemaker implantation or elective unit replacement. Heart 1998; 80: 240-4.

14. Pavia S, Wilkoff B. The management of surgical complications of pacemaker and implantable cardioverter-defibrillators. Curr Opin Cardiol 2001; 16: 66-71.

15. Sohail MR, Uslan DZ, Khan AH, Friedman PA, Hayes DL, Wilson WR, et al. Management and outcome of permanent pacemaker and implantable cardioverter-defibrillator infections. J Am Coll Cardiol 2007; 49: 1851-9. 